\title{
A simple deterministic self-organized critical system
}

\author{
Maria de Sousa Vieirat \\ Department of Biochemistry and Biophysics, University of California, San Francisco, California 94143-0448.
}

\begin{abstract}
We introduce a new continuous cellular automaton that presents self-organized criticality. It is one-dimensional, totally deterministic, without any embedded randomness, not even in the initial conditions. This system is in the same universality class as the Oslo rice pile, boundary driven interface depinning and the train model for earthquakes. Although the system is chaotic, in the thermodynamic limit chaos occurs only in a microscopic level.
\end{abstract}

In 1987, Bak, Tang and Wiesenfeld showed that fractal behavior, that is, power-law distributions, can be observed in simple dissipative systems with many degrees of freedom without fine tuning of parameters [1]. They called this phenomenon self-organized criticality (SOC). Until then, the studies of fractal structures were basically related to equilibrium systems where fractality appears only at special parameter values where a phase transition takes place.

Since the pioneering work of Bak et. al, an enormous amount of numerical, theoretical and experimental studies have been done in systems that present SOC. One of the most interesting experimental studies demonstrating the existence of SOC in Nature was done in a quasi-onedimensional pile of rice by Frette et. al [2]. They found that the occurrence of SOC depends on the shape of the rice. Only with sufficient elongated grains, avalanches with a power-law distribution occurred. If the rice had little asymmetry, a distribution described by a stretched exponential was seen. Christensen et. al [3] introduced a model for the rice pile experiment in which the local critical slope varies randomly between 1 and 2 . They found that their model, known as the Oslo rice pile model, reproduced well the experimental results.

A good understanding of the Oslo system was achieved by Paczuski and Boettcher [4]. They showed that it could be mapped exactly to a model for interface depinning where the interface is slowly pulled at one end through a medium with quenched random pinning forces. They found that the height of the interface maps to the number of toppling events in the rice pile model. The critical exponents of the two models were identical (within the error bars), showing that they were in the same universality class. Paczuski and Boettcher also conjectured that the train model for earthquakes, which was introduced by Burridge and Knopoff [5], and studied in detail in [6], is also in that same universality class. The train model is the only model that we know (besides the one we introduce here) that presents SOC and has no kind of embedded randomness. However, it is governed by coupled ordinary equations (ODE's), what makes its study very time consuming.

A way of making a system governed by ODE's more amenable to computer simulations is to discretize it in time. This was done by Olami, Feder and Christensen (OFC) [7] who introduced a continuous cellular automaton (CCA) to study the two-dimensional version of another Burridge and Knopoff model for earthquakes [5]. [A continuous cellular automaton in SOC is known in chaos theory as coupled lattice maps. These systems are characterized as having space and time variables defined in the domain of real and integer numbers, respectively.] In the OFC model, SOC is seen only in systems that have a geometry with dimensionality of at least two. That system is a variation of a model introduced by Nakanishi [8], which has a one-dimensional geometry. However, the model introduced by Nakanishi does not present SOC, since the power-law distribution it presents has an upper cutoff that is unrelated to the system size.

Here we introduce a new self-organized critical system, that is governed by a CCA (that is, the space is continuous and the time is discrete). It is one-dimensional and has no embedded randomness, not even in the initial conditions. We will show that our model belongs to the same universality class as the Oslo rice pile, boundary driven interface depinning and the train model. The importance of our results comes from the fact that we show that it is possible to map stochastic SOC systems to simple, discrete, chaotic systems, in which no randomness exists. Such an equivalence of a deterministic model with no embedded randomness which is chaotic with a stochastic model also occurs between the deterministic KuramotoShivashinsky [10] equation and the Langevin equation proposed by Kardar, Parisi and Zhang [11]. In our opinion, the train model governed by ODE's already achieved this [6]. However, because its simulation is very time consuming, it will probably be impossible to find such equivalences for higher dimensional (two, three, etc.) systems, unless the system is discretized in time, as we do here. In fact, we are unaware of any studies on train-like systems with dimensionality higher than one. To the best of our knowledge, our model is the only SOC system introduced so far that is one-dimensional, totally deterministic and with discrete time.

Another important result of this paper concerns to the fact that we show that although chaos exists in the model, it decreases as the system size increases, and in thermodynamic limit it exists only in a microscopic level. Con- 
sequently, our results indicate that the fractal structures seen in Nature and supposed associated with SOC, could in principle result only from nonlinearities in those systems, without any need for the presence of random irregularities. Such fundamental questions are also found in equilibrium statistical mechanics, where it is unknown if probability theory is only a tool to describe phenomena that in principle could have been originated solely from microscopic chaos [12].

The train model is shown in Fig. 11(a). It consists of a chain of blocks connect by harmonic springs. The blocks are on a rough surface with friction, and the first block is pulled slowly with a constant velocity by a driver. The dynamics of the model is as follows: suppose that all the blocks are initially at rest. As the driver pulls the first block, it remains stuck until the elastic force overcomes the static friction. When this occurs, the first block will move a little and will be stopped again by friction. Such small events (or earthquakes) will continue, and will increase the elastic force on the second block. There will be a moment when the elastic force on the second block will overcome the friction force, and then we will see an event involving two blocks. This kind of dynamics will continue with events involving three, four, or all the blocks in the system.

The elastic force in block $i$ is given by $f_{i}=x_{i-1}-$ $2 x_{i}+x_{i+1}$, where $x_{i}$ is the displacement of block $i$ with respect to its equilibrium position (without losing generality, the spring constant can be taken as equal to 1 [6]). The boundary conditions are $x_{0}=0$ and $x_{L+1}=x_{L}$. After an earthquake, in which block $i$ was displaced by $\Delta x$, the elastic forces in block $i$ and in its nearest neighbors will be $f_{i}^{\prime}=f_{i}-\Delta f$, and $f_{i \pm 1}^{\prime}=f_{i \pm 1}+\Delta f / 2$, respectively, where $\Delta f=2 \Delta x$. Thus, the force that is relaxed in block $i$ is redistributed equally to its near nearest neighbors, implying conservation of elastic forces. This is embedded into the geometry of the system. However, the model does dissipate energy through friction between the rough surface and the blocks. Consequently, the model is conservative with respect to elastic forces, and dissipative with respect to energy. This is one of the main distinctions between the train model and the other Burridge and Knopoff earthquake model studied by Nakanishi and OFC, in which neither the energy nor the forces are conserved.

In the discretized version of the train model that we introduce here, each block $i$ is characterized by a variable $f_{i}$, which we will call force, with $i=1, \ldots, L$, and $L$ being the number of blocks in the system. The boundary conditions are the same as the ones in the train model, which are given above. The dynamical evolution of the system is determined by the following algorithm:

(1) Start the system by defining initial values for the variables $f_{i}$, which can be the same for all the blocks, so the they are below a chosen, fixed, threshold $f_{t h}$.

(2) Update the force in the first block by incrementing its force to the threshold value plus a fixed small value $\delta f$, i.e., $f_{1}=f_{t h}+\delta f$ (an event is going to be triggered).

(3) Check the forces in each block. If a block $i$ has $f_{i} \geq f_{t h}$, update $f_{i}$ according to $f_{i}^{\prime}=\phi\left(f_{i}-f_{t h}\right)$, where $\phi$ is a given nonlinear function that has a parameter $a$. Increase the forces in its two nearest neighboring blocks according to $f_{i \pm 1}^{\prime}=f_{i \pm 1}+\Delta f / 2$, where $\Delta f=f_{i}-f_{i}^{\prime}$.

(4) If $f_{i}^{\prime}<f_{t h}$ for all the blocks, go to step (2) (the event has finished). Otherwise, go to step (3) (the event is still evolving).

One can use either parallel or sequential update in the evolution of the system. We have verified that the critical exponents of the model do not depend on the type of update chosen. The systems is governed by $L$ variables and has two parameters, $a$ and $\delta f$, since without losing generality we can take $f_{t h}=1$. The force in our model is supposed to mimic the combination of two forces in the train model, that is, the elastic and the friction forces. The elastic force is periodic, whereas the friction force in simulations is generally assumed to decrease with the velocity of the block. We have found numerically that $\phi$ mimics the combination of these two force when it is a periodic function, since only in this way the system presents SOC behavior. So, the periodicity of the elastic force dominates over the form of the friction force. The models introduced by Nakanishi and OFC assume that $\phi$ is a strictly nonincreasing function. We have found that if we use a strictly nonincreasing function for $\phi$, such as the one used in [8], we observe in our model the presence of stretched exponentials, instead of powerlaws [9]. It is worth noting that in this situation our model reproduces the results of the distributions found with nearly round rice in [2], which were also governed by stretched exponentials.

The functional form we chose for $\phi$ is shown in Fig. 1(b), which is given by, $\phi(x)=1-a[x]$ where $[x]$ denotes $x$ modulo $1 / a$, that is, a sawtooth function. However, we have tested several other periodic functions, and found that the SOC behavior we show here remains, that is, the results are robust, the essential ingredient being periodicity (not necessary a perfect one) for $\phi$.

In Fig. 2 we show the distribution of events involving $s$ update steps, which is the size of the event, using parallel update. The events that involve all the blocks of the chain have been excluded from our analysis, since they do not belong to the same distribution, as expected. Before we start to compute the statistics, we wait until the last block has moved, in order to neglect transient effects. In (a) we show three different cases, with $L=512$, in which we have varied one parameter at a time. The solid line refers to $a=4$ and $\delta f=0.1$, the dashed line is for $a=4$ and $\delta f=0.01$, and the short-dashed line refers to $a=2.5$ and $\delta f=0.01$. We see that the small events have their own distribution, like in the Oslo model for rice piles [3]. A careful analysis has shown that these small events have an exponential distribution [9]. As $s$ 
is increased the distribution becomes a power-law, which has a cutoff related to finite size effects, only. We find that the slope of the power law is independent of $a$ and $\delta f$. However, the crossover point $s^{*}$ of the exponential behavior to the power law one depends on $a$, but not on $\delta f$. The frequency of the small events is inversely proportional to $\delta f$.

In Fig. 2(b) we show simulations keeping $a$ and $\delta f$ fixed, with $a=4$ and $\delta f=0.1$, and varying $L$. We see that increasing $L$, the range of the power-law increases. To fit the data to a single curve, we try the finite size scaling ansatz $P(s, L)=s^{-\tau} G\left(s / L^{D}\right)$, where $D$ and $\tau$ are the basic exponents of the model [13], defining its universality class. $D$ and $\tau$ are called the dimension and the size distribution exponents, respectively. In our model we find that $\langle s\rangle \sim L$ which results in $\tau=2-1 / D$. The best fit for $P(s, L)$ is found for $\tau \approx 1.54$ and $D \approx 2.20$. The data collapse for these values of the exponents is shown in Fig. 2(c). Within the error bars, these exponents are the same ones of the Oslo rice pile [2, 过, driven boundary interface depinning 迎 and the train model for earthquake [4.9. Consequently, all these models, including the one we introduce here, are in the same universality class.

In Fig. 3(a) we show the frequency of the events $P(T)$ as a function of the its time duration $T$, for different system sizes. The parameters are the same as in Fig. 2(b). Again, we see a power-law distribution, except for the smallest events. A data collapse for the function $P(T, L)=T^{-y} f_{T}\left(T / L^{\sigma}\right)$ is shown in Fig. 3(b), with $y=1.84$ and $\sigma=1.40$. These are the same exponents found in the Oslo rice pile. From conservation of probability it follows that $\sigma(y-1)=D(\tau-1)$ in good agreement with our results. The results shown in Fig. 3 are, again, for parallel update, since in sequential update the time duration and event size coincide resulting in $y=\tau$ and $\sigma=D$.

Using the method introduced by Benettin et al. 14, we have calculated the largest Liapunov exponent, $\lambda_{1}$, and the second largest Liapunov exponent, $\lambda_{2}$, of the system. If $\lambda_{1}$ is greater than zero, it implies that the system has a strong sensitivity to the initial conditions, and by definition, it is called chaotic. To study the Liapunov exponents we have chosen sequential update. The reason for this is that the calculation of the Liapunov exponent assumes, by its own definition, that small changes happen in the system in the time unit, and this is more consistent with sequential rather than with parallel update. In Fig. 1 (a) and Fig. 团(b) we show $\lambda_{1}$ and $\lambda_{2}$ as a function of $a$ for $\delta f=0.1$, and as a function of $\delta f$ for $a=4$, respectively. In both cases $L=64$. We have found that for $a \leq 1$ the system is in continuous motion, and therefore being impossible to define earthquakes. Consequently, SOC is only seen when $a>1$. We see that the Liapunov exponents increase as $a$ or $\delta f$ increases, with the other parameters kept fixed. Fig. 1(c) shows the largest Liapunov exponent as a function of the sys- tem size for $a=4$ and $\delta f=0.1$ (solid), $a=4$ and $\delta f=0.01$ (dashed) and $a=2.5$ and $\delta f=0.01$ (shortdashed). We observe that $\lambda_{1}$ is approximately constant for small $L$ and decreases nearly as power-law when $L$ is greater than a certain value. The value where this bending occurs seems to be sensitive to both $\delta f$ and $a$. Since $\lambda_{1} \rightarrow 0$ in the thermodynamic limit $(L \rightarrow \infty)$ we conclude that chaos exists only in a microscopic level, and that any time or space scales in the system are negligible. We have studied the system using slower time scales, such as measuring time by the updates in the first block. Still we find that $\lambda_{1} \rightarrow 0$ when $L \rightarrow \infty$. In the train model governed by ODE's and pulled with a constant finite velocity we have found that the largest Liapunov exponent tends to a constant as the system size increases [15]. However, our new unpublished results shows that the Liapunov exponents in that system start to decrease for system sizes greater than a given value, as it happens in the system we introduce here.

* $\quad$ Electronic address: mariav@msg.ucsf.edu.

[1] P. Bak, C. Tang, and K. Wiesenfeld, Phys. Rev. Lett. 59, 381 (1987); P. Bak et. al, Phys. Rev. A 38, 364 (1988).

[2] V. Frette et. al, Nature (London) 379, 49 (1996).

[3] K. Christensen et. al, Phys. Rev. Lett. 77, 107 (1996).

[4] M. Paczuski and S. Boettcher, Phys. Rev. Lett. 77, 111 (1996).

[5] R. Burridge and L. Knopoff, Bull. Seismol. Soc. Am. 57, 341 (1967).

[6] M. de Sousa Vieira, Phys. Rev. A 46, 6288 (1992).

[7] Z. Olami et. al, Phys. Rev. Lett. 8, 1244 (1992).

[8] H. Nakanishi, Phys. Rev. A 43, 6613 (1991).

[9] M. de Sousa Vieira, to be published.

[10] V. Yakhot, Phys. Rev. A 24, 642 (1981).

[11] M. Kardar, G. Parisi, and Y.-C Zhang, Phys. Rev. Lett. 56889 (1986).

[12] G. M. Zaslavsky, Phys. Today 52, 39 (1999).

[13] M. Paczuski, S. Maslov and P. Bak, Phys. Rev. E 53, 414 (1996).

[14] G. Benettin et al., Phys. Rev. A 14, 2338 (1976).

[15] M. de Sousa Vieira et. al., Phys. Rev. E 53, 1441 (1996).

FIG. 1. (a) The train model. (b) The function $\phi(x)$.

FIG. 2. Probability distribution $P(s)$ of the number of toppling events $s$. (a) solid: $L=512, a=4, \delta f=0.1$; dashed: $L=512, a=4, \delta f=0.01$; short-dashed: $L=512$, $a=2.5, \delta f=0.01$. (b) $L=32,64,128,256,512$ with $a=4$ and $\delta f=0.1$. (c) Data collapse of the cases shown in (b) with $\tau=1.54$ and $D=2.20$. 
FIG. 3. (a) Probability distribution $P(T)$ of the number of toppling events $T$ for $L=32,64,128,256,512$ with $a=4$ and $\delta f=0.1$. (b) Data collapse of the cases shown in (a) using $P(T)=T^{-y} f_{T}\left(T / L^{\sigma}\right)$ with $y=1.84$ and $\sigma=1.40$.

FIG. 4. (a) The largest (solid) and the second largest (dashed) Liapunov exponents as a function of $a$ for $\delta f=0.1$, and (b) as a function of $\delta f$ for $a=4$, with $L=64$ in both cases. (c) The largest Liapunov exponent as a function of $L$ for $a=4$ and $\delta f=0.01$ (dashed), $a=2.5$ and $\delta f=0.01$ (short-dashed). The error bars associated with this calculation have approximately the size of the plus sign shown on the curves. 
Fig. 1

(a)
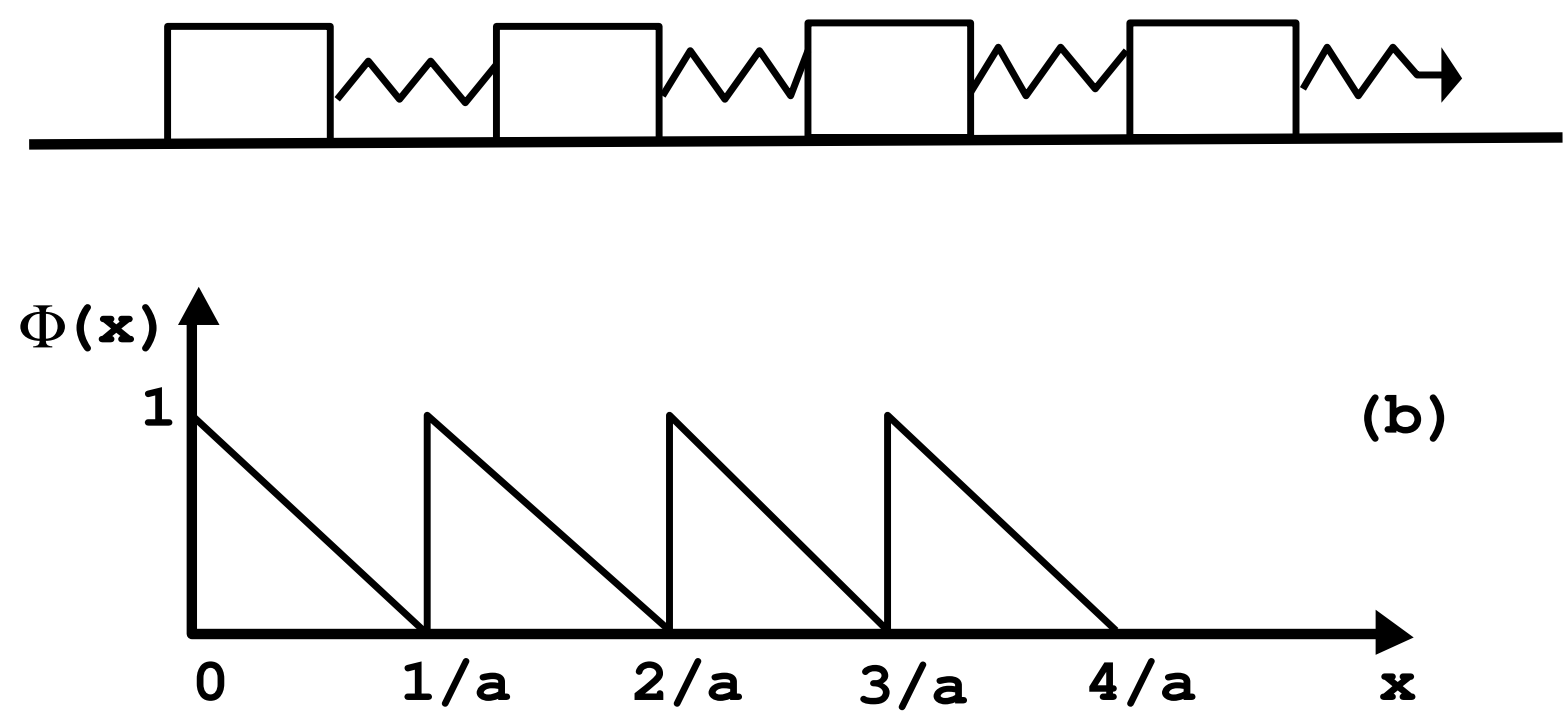
Fig. 2(a)

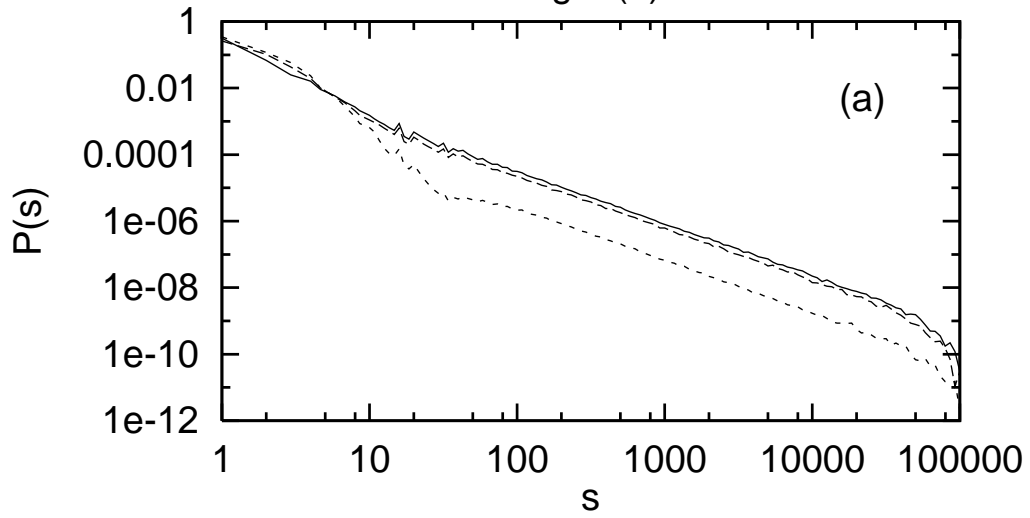

Fig. 2(b)

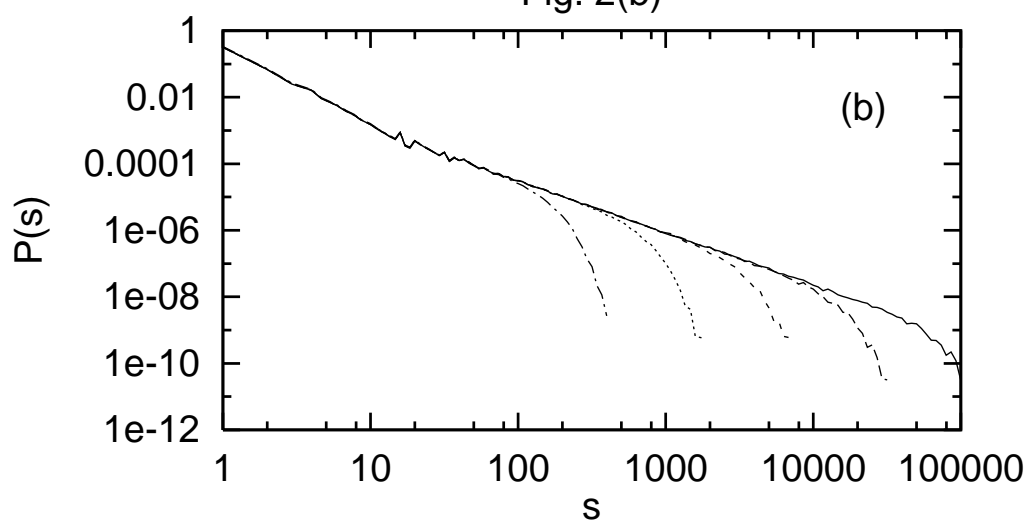

Fig. 2(c)

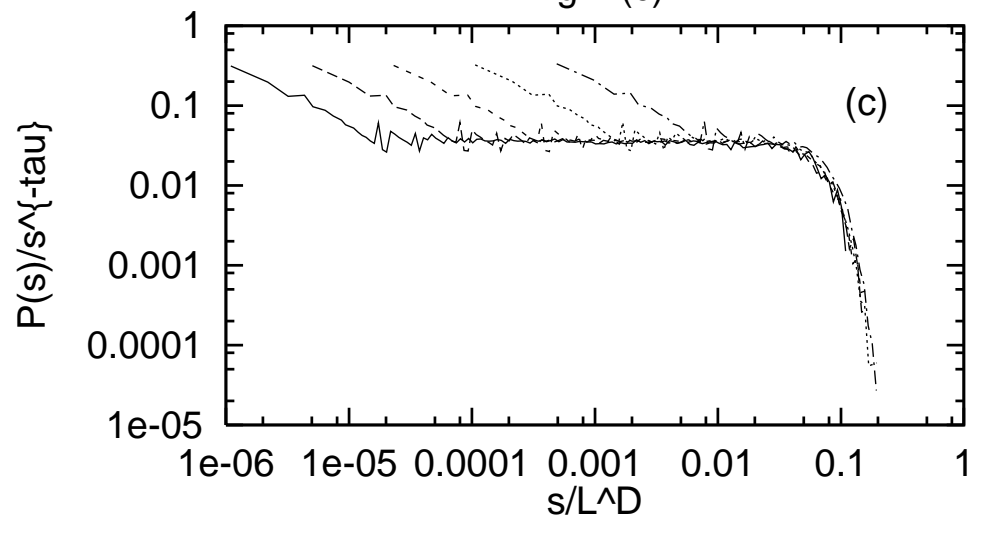


Fig. 3(a)

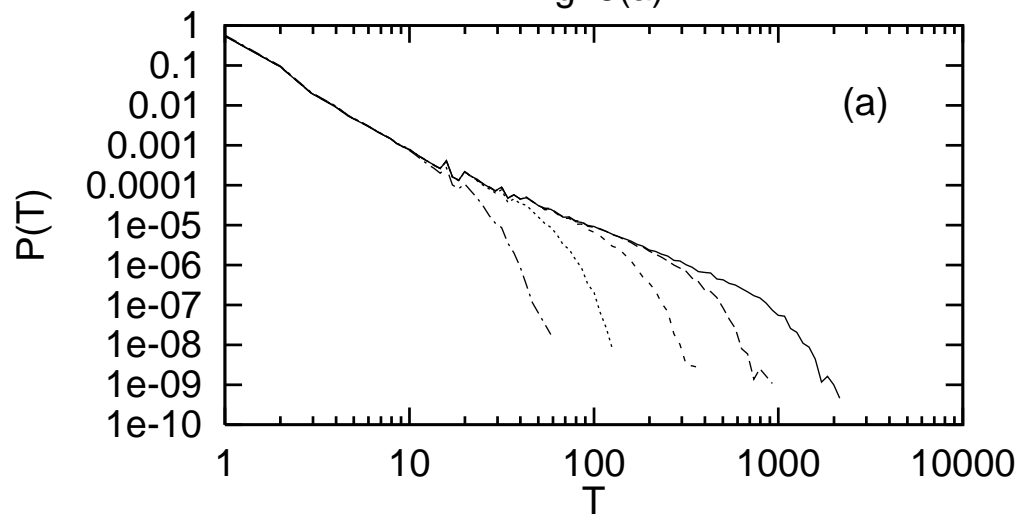

Fig. 3(b)

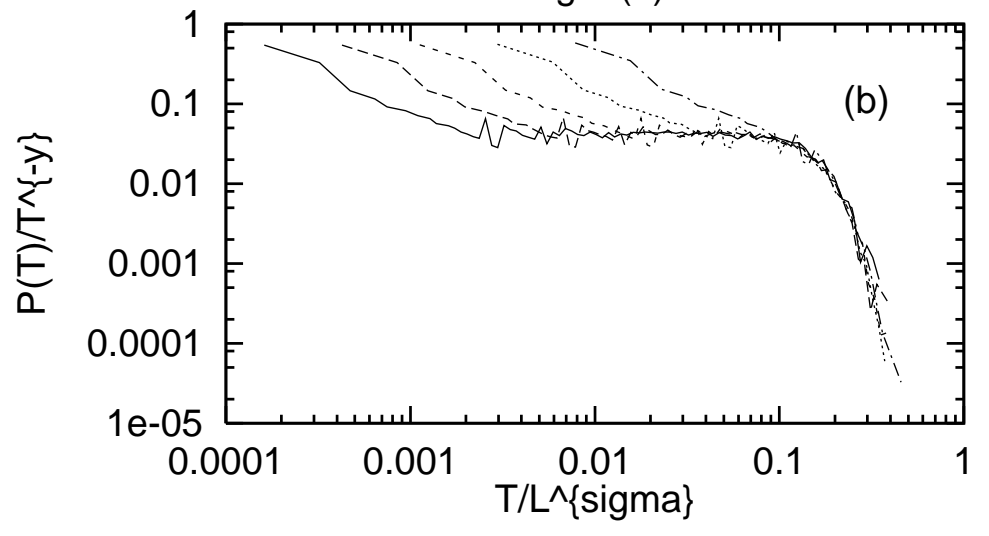


Fig. 4(a)

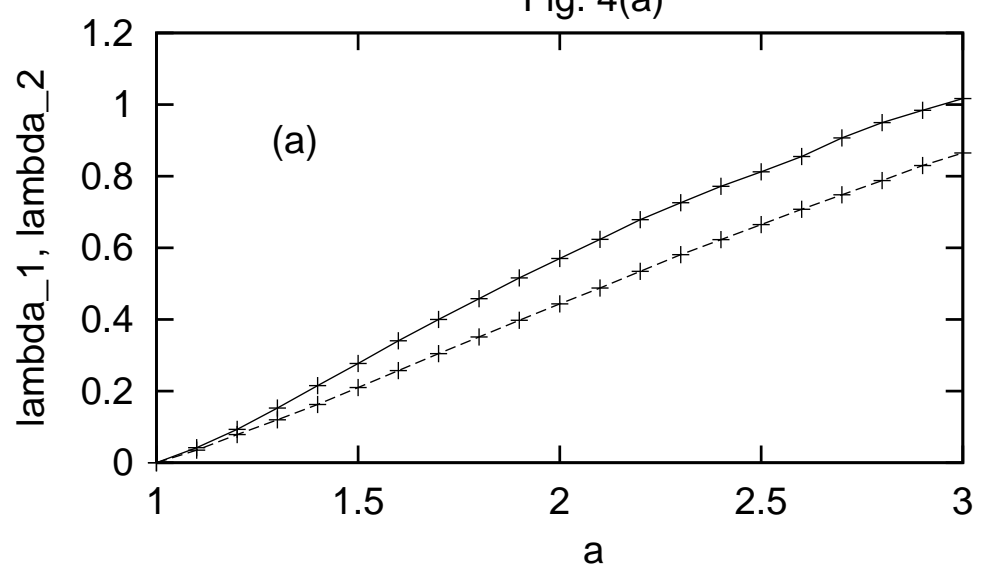

Fig. 4(b)

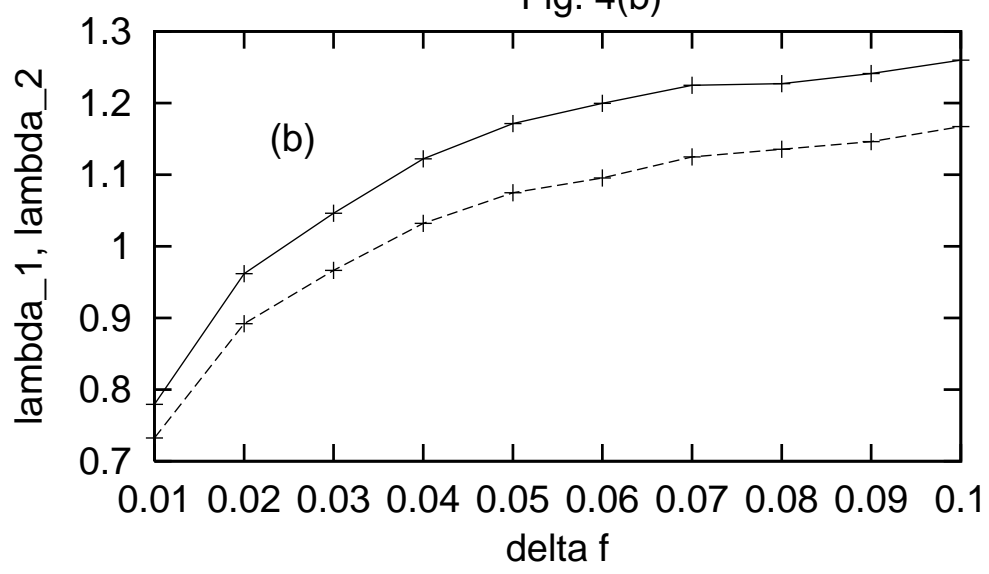

Fig. 4(c)

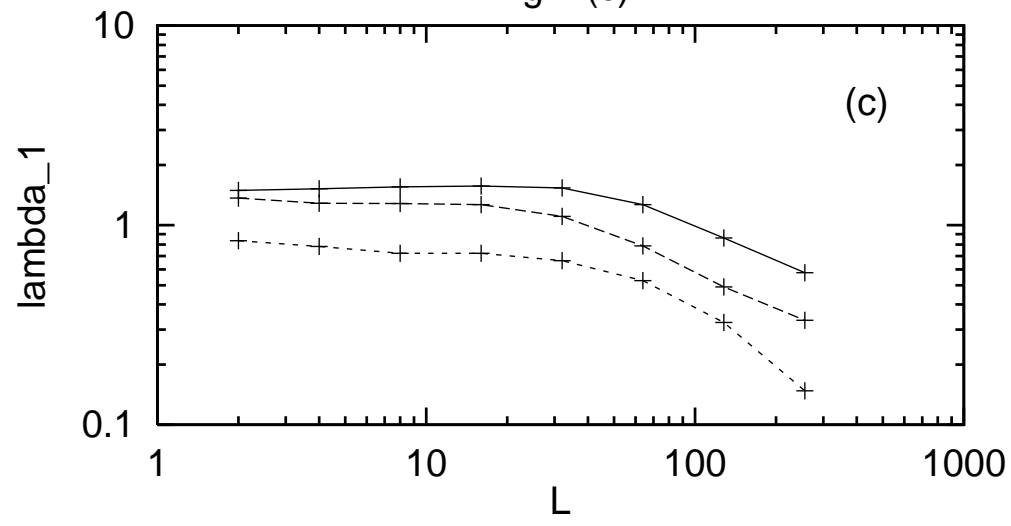

